\title{
Acute effects of static stretching of upper arm and forearm on the accuracy of free throws in basketball
}

\author{
Igor Vučković ${ }^{1}$ and Aleksandar Gadžić ${ }^{2, *}$ \\ ${ }^{I}$ Faculty of Physical Education and Sport, University of Banja Luka, Banja Luka, Bosnia and Herzegovina; and ${ }^{2}$ Faculty \\ of Physical Culture and Sports Management, Singidunum University, Belgrade, Serbia
}

Copyright: @ 2016 I. Vučković and A. Gadžić. This is an open access article licensed under the Creative Commons Attribution License (http://creativecommons.org/licenses/by/4.0/).

\begin{abstract}
Background: Free throws are a very important element in modern basketball. There are many studies focused on different aspects ranging from variability in the mechanics of throwing at various skill levels to the influence of free throws on the results of games. Objective: Since the authors of the present study noticed that some players during a game apply static stretching of shooting arm as a preparation to perform the free throw, we wanted to question the justification of such procedure prior to the performance of free throws. The purpose of this study was to determine an effect of submaximal 15 seconds static stretching of the upper arm (m. triceps brachii) and forearm (m. flexor carpi radialis) on the accuracy of free throws in basketball. Methods: Participants of the present study were 100 basketball players; mean age was $18.2 \pm 2.54$ years. The sample was divided into two groups - players with higher $(n=70 ; 6.83 \pm 1.83$ hours/week) and lower number $(n=30 ; 4.17 \pm 0.48$ hours/week $)$ of training sessions per week. Furthermore, we compared separately experienced ( $n=47 ; 12.2 \pm 0.55$ years) and less experienced basketball players $(n=53 ; 6.4 \pm 0.45$ years). The procedure purported that every player performs five free throws without prior stretching, five free throws immediately after 15 seconds of static stretching of upper arm muscle (m. triceps brachii), and five free throws after 15 seconds of static stretching of the forearm (m. flexor carpi radialis). Results: Fifteen seconds of static stretching of the upper arm and forearm muscles had a negative influence on the accuracy of free throw shooting in basketball. Players with a higher number of training sessions per week showed better tolerance to this negative influence. Conclusion: Results of this study indicate that is not useful to apply static stretching of the triceps brachii and flexor carpi radialis muscles just before the execution of free throws.
\end{abstract}

Keywords: basketball, triceps brachii, flexor carpi radialis, stretching

\section{Introduction}

Basketball is full of technical and tactical elements. However, we should bear in mind that children start playing basketball in order to shoot at the hoop, score a point, and compete with the opponent and themselves. That is why shooting at the hoop, as one of the most important elements of basketball technique, is a special challenge for the coach. One of the very important ways to shoot at the hoop is a free throw. Players perform many free throws in a game and that segment of the offensive game very often decides the winner (Zuzik, 2011). Karalejić and Jakovljević (2001) claim

\footnotetext{
* Address for correspondence: Aleksandar Gadžić, Department of Physical Education, Faculty of Physical Culture and Sports Management, Singidunum University, Danijelova 32, 11000 Belgrade, Serbia. E-mail: agadzic@singidunum.ac.rs
}

that success in free throws, besides throwing mechanics, is under the influence of - performance routine, relaxation, self-esteem, and concentration. There are studies that focused on relationships between psychological traits of basketball players and their successful throws percentage (Foster, Weigand, \& Baines, 2006; Schmidt, 2012; Wilson, Vine, \& Wood, 2009). Furthermore, authors investigated the influence of fatigue on free throw accuracy (Mah, Mah, Kezirian, \& Dement, 2011; Memmert, 2006; Woolstenhulme, Bailey, \& Allsen, 2004). Other authors tried to find optimal biomechanical parameters for successful performance of free throws (Button, Macleod, Sanders, \& Coleman, 2003; Mullineaux \& Uhl, 2010). There are authors that investigated the influence that free throws have on the final results of games (Csataljay, O'Donoghue, Hughes, \& Dancs, 2009; Navarro, Lorenzo, Gómez, \& Sampaio, 2009; Sampaio, Lorenzo, \& Ribeiro 2006). 
Shooting accuracy was defined as the basketball players ability to hit the hoop with precisely directed and measured ball throw (Perić \& Petrović, 2015). This ability is mostly congenital, but with proper technique and a large number of repetitions it could be improved (Malacko, 2000). Furthermore, it has been known, for quite some time, that successful movement execution is directly linked with adequate feedback (Bilodeau, Bilodeau, \& Schumsky, 1959).

On the other hand, it is considered that flexibility of muscles and joints is an important part of basketball players' fitness. Today, flexibility exercises are an integral part of every warm-up (dynamic exercises), and cooling down in training (static exercises). Stretching is usually performed before exercise in an attempt to enhance performance and reduce the risk of injury (Weerapong, Hume, \& Kolt, 2004).

Stojanović, Stojanović, Veljović, Međedović, \& Ostojić (2009) wrote:

The exact mechanism of neural aspects of muscletendon unit stretching is still unknown. It seems the increase of movement amplitude is mostly defined by the improvement of tolerance on the performed stretching. If the targeted muscle is stretched during static stretching, the resistance of stretching, defined as momentum in joint will decrease with time elapsed. (p. 68)

The usual warm-up routine consists of: slow running with and without the ball (cca $8 \mathrm{~min}$ ), dynamic warm-up and stretching exercises (cca $8 \mathrm{~min}$ ), layups and shooting (cca $10 \mathrm{~min}$ ), then free throws and team announcements (cca $6 \mathrm{~min}$ ). It is quite clear that within last 16 minutes all potential effects of stretching on free throws performance would disappear once the game starts. However, we noticed that some players during a game apply static stretching of shooting arm as a preparation to perform the free throw. Considering that, we wanted to question the justification of such procedure prior to the performance of free throws.

Anatomically speaking, the muscles that lift and throw the ball to the hoop are triceps brachii, deltoideus, flexor carpi radialis and biceps brachii. In that exact order, the movement contribution of these muscles was established by electromyography of free throws performance (Zachry, Wulf, Mercer, \& Bezodis, 2005). Deltoideus and biceps brachii muscles lift the ball, the throwing movement is performed by triceps brachii and $\mathrm{m}$. flexor carpi radialis muscle (Bošković, 1990). Conforming to that fact, triceps brachii and flexor carpi radialis muscles of the shooting arm were statically stretched just before the performance of the free throws.
Based on our experience with junior players, we noticed that some of them use brief static stretching of arms prior to the free throws execution. We wanted to assess the suitability of this practice. There is firm evidence of reduced sports performance immediately after stretching exercise (Cramer et al., 2004; Kay \& Blazevich, 2008; Knudson \& Noffal, 2005; Stojanović et al., 2009). That is why we assumed that $15 \mathrm{~s}$ of the upper arm and forearm muscles stretching would have a negative impact on the shooting accuracy, thence to lower percentage of free throws. If our assumptions are right, it might be useful for coaches to teach their players to avoid this sort of activity before shooting free throws, in practice and/or game situations.

\section{Methods}

\section{Participants}

A hundred junior and senior basketball players participated in this study. The mean age of participants was $19.32 \pm 3.67$ years. There were 53 junior players (age ranged from 15 to 18 years; mean $16.66 \pm 0.83$ years) who compete at youth league of Republic of Srpska, and 47 senior players ranging from 19 to 34 years of age $(22.32 \pm 3.28 ; 13$ compete at international level - Adriatic Basketball Association League, and 34 compete at second division of Bosnia and Herzegovina). Based on the amount of training hours per week $(6.03 \pm 1.98$ hours/week), the participants were divided into a group with 6 and more hours per week ( $n=70 ; 6.83 \pm 1.83$ hours/week) and until five hours ( $n=30 ; 4.17 \pm 0.48$ hours/week) groups. We analyzed the significance of differences between these two groups and its potential influence on free throws accuracy. For observation of the potential influence of the overall basketball experience on free throws accuracy, for the analysis purpose, based on mean experience $(9.5 \pm 2.0$ years), we divided the sample into groups of over 10 years $(n=47 ; 12.2 \pm 0.55$ years $)$ and below 10 years ( $n=53 ; 6.4 \pm 0.45$ years) of experience.

All procedures were conducted in accordance with the ethical standards of the Helsinki Declaration and all the participants gave their written informed consent.

\section{Procedures}

Without a prior warm-up, the basketball player stands on the free throw line, takes the ball, and performs two trial free throws that do not count in the score. After that, he shoots five free throws. The score taker writes down the score. Then, at the timekeeper's signal, the player performs stretching of muscle triceps brachii of shooting arm for $15 \mathrm{~s}$ (Figure 1A). Following that, he executes five free throws, which count in the 

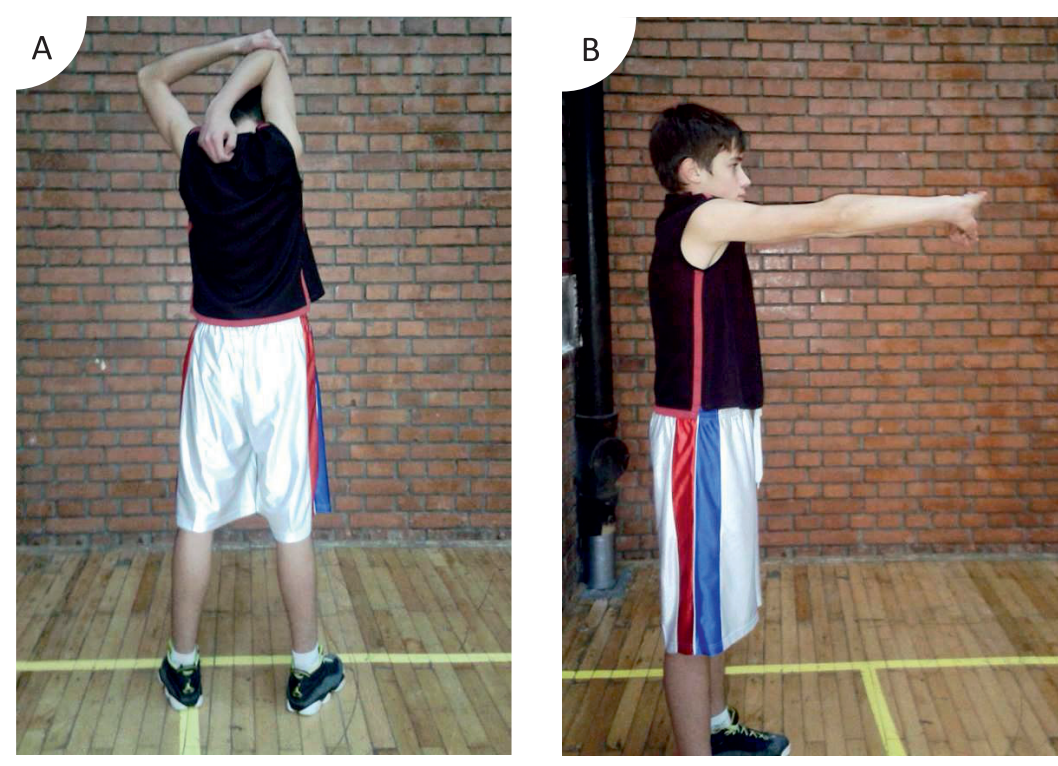

Figure 1. Stretching of triceps brachii (A) and flexor carpi radialis (B).

score. Furthermore, at the time keeper's signal starts to stretch forearm muscles (flexor carpi radialis) for $15 \mathrm{~s}$ (Figure 1B). After that, he performs five free throws. Applied intensity of stretching was submaximal (submaximal stretching intensities; POD-point of discomfort, as recommended in Chaouachi et al., 2010).

The previous warm-up was eliminated due to a possible unequal influence on the participants and their accuracy - especially if arms stretching have been done. Two "trial" free throws were enlisted in the protocol to "equal starting positions". It means that some teams might have training session 6 hours before the testing, and second one 48 hours. It was not allowed to tap the ball between the attempts, but a correction in the arm was permitted. All of the tests were conducted in a sports hall, during the competitive season, before the usual training session. A stopwatch was used to take time of the performed stretching. Free throws were performed with a Spalding TF500 size 7 basket ball.

\section{Statistical analyses}

Microsoft Office Excel (Version 2007; Microsoft, Redmond, WA, USA) and SPSS (Version 20; SPSS, Chicago, IL, USA) were used to process the data. Percentages of successful free throws were calculated. We used $\chi^{2}$ test to determine differences between fifth and sixth series, and between tenth and eleventh series of free throws. Mann-Whitney $U$ test was applied to determine differences in terms of basketball experience and training sessions per week.

\section{Results}

The results of the present study were presented within five graphs. We tried to follow the tendency of free throws performance from the first to the fifth throw in all of the three series of throws. Special attention was given to the sixth and eleventh free throw (those were the first ones after the stretching).

As presented in Figure 2, from the first to the fifth free throw success showed an increasing trend, except for the fourth throw. After the $15 \mathrm{~s}$ of static stretching of the triceps brachii muscle, the percentage of successful free throws decreases (from initial $79 \%$ to $\left.68 \% ; \chi^{2}=3.11 ; d f=1 ; p=.08\right)$. The second and the third free throw were realized in increasing percentage while fourth and fifth had a slight decline in success

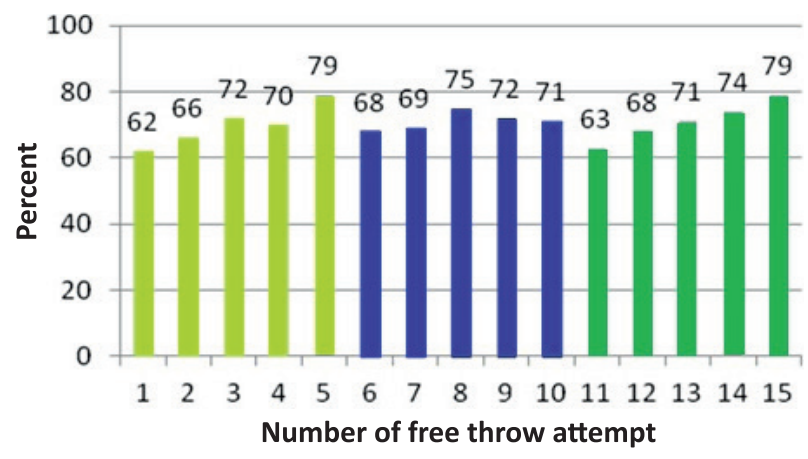

Figure 2. Percentage of the free throws before the stretching (columns 1-5), after the stretching of triceps brachii muscle (columns 6-10), and after the stretching of flexor carpi radialis muscle (columns 11-15) for the whole sample $(N=100)$. 
percentage. After the second round of static stretching (m. flexor carpi radialis), a notable drop in the success percentage of the first free throw (from $71 \%$ to $63 \%$; $\left.\chi^{2}=1.45 ; d f=1 ; p=.23\right)$, but every succeeding shot added on to the growing tendency of the percentage success.

The basketball players of the below average training sessions per week group $(n=30)$ have a lower percentage of successful free throws (68\%), and more irregular tendency of scored throws after stretching (Figure 3). In the above average training sessions per week group (percentage of success $=72 \%$ ), it is an obvious increasing tendency of successful free throws after the second round of stretching (Figure 4). Mann-Whitney $U$ test has shown there are no differences between the players with higher and lower number of training sessions per week in any series of free throws performed.

Considering all of the 15 free throw attempts, basketball players with below average experience have the

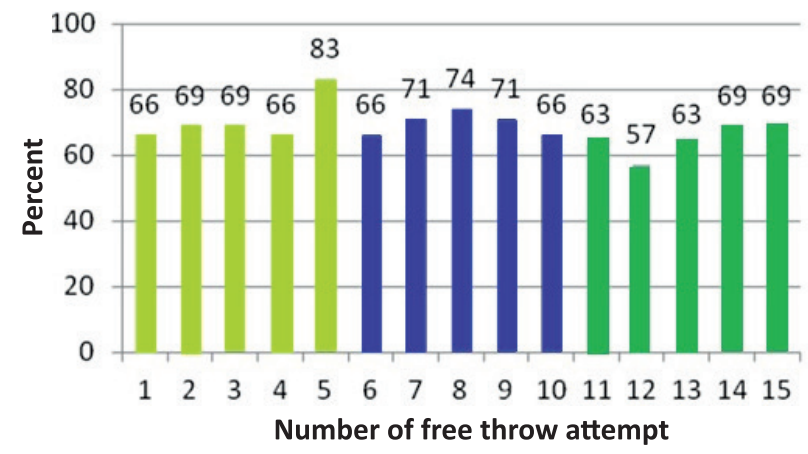

Figure 3. Percentage of the free throws before the stretching (columns 1-5), after the stretching of triceps brachii muscle (columns 6-10), and after the stretching of flexor carpi radialis muscle (columns 11-15) for the group of below average training sessions per week $(n=30)$.

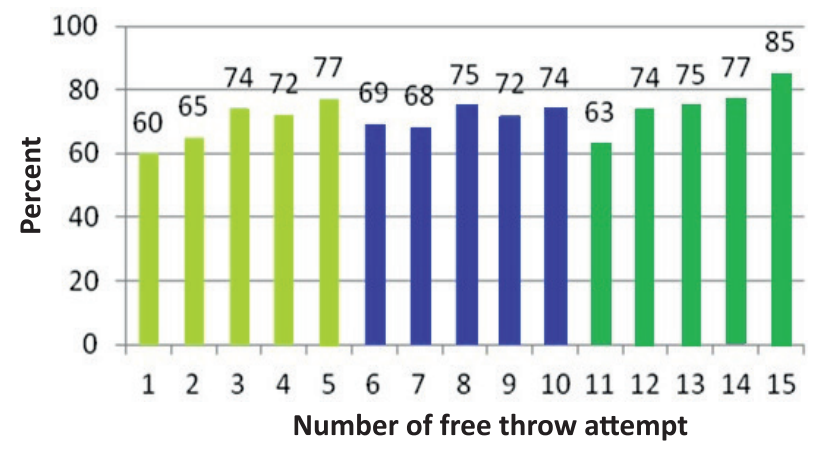

Figure 4. Percentage of the free throws before the stretching (columns 1-5), after the stretching of triceps brachii muscle (columns 6-10), and after the stretching of flexor carpi radialis muscle (columns 11-15) for the group of above average training sessions per week $(n=70)$. same percentage of successful free throws $(70.5 \%)$ as players with above average experience. Within both groups, scoring tendency before the stretching was increasing rather regularly, but after the stretching, that tendency is not clear. However, the percentage of the first free throw performed after the stretching (sixth and eleventh throw) in both groups is lower than in previously performed throw (Figures 5 and 6). Although these decreases in percentages were not statistically significant $\left(\chi^{2}=3.11 ; d f=1 ; p=.08\right)$, our results suggest a negative impact of that stretching on the shooting accuracy for less experienced players after both stretching procedures.

\section{Discussion}

The main objective of this study was to investigate the influence of $15 \mathrm{~s}$ static stretching of upper arm muscle

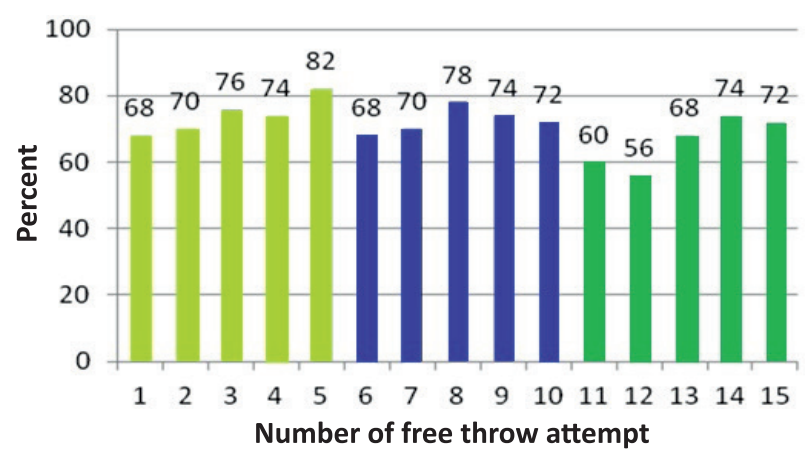

Figure 5. Percentage of the free throws before the stretching (columns 1-5), after the stretching of triceps brachii muscle (columns 6-10), and after the stretching of flexor carpi radialis muscle (columns 11-15) for basketball players with the below average experience $(n=53)$.

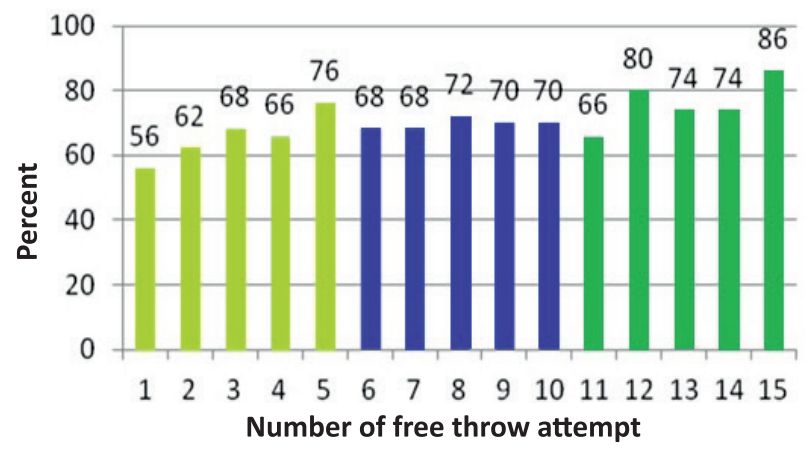

Figure 6. Percentage of the free throws before the stretching (columns 1-5), after the stretching of triceps brachii muscle (columns 6-10), and after the stretching of flexor carpi radialis muscle (columns 11-15) for basketball players with the above average experience $(n=47)$. 
(triceps brachii) and forearm muscle (flexor carpi radialis) on the accuracy of free throws in basketball. Since previous studies confirmed that static stretching techniques decrease neuromuscular reflex sensitivity and neural inhibition (Avela, Kyrolainen, \& Komi, 1999; Ribot-Ciscar, Tardy-Gervet, Vedel, \& Roll, 1991; Vujnovich \& Dawson, 1994), it was presumed that both stretching procedures would have a negative influence on the free throw accuracy. The results shown in Figure 2 led to two key points. Firstly, there is a noticeable, regular increasing trend of successful free throws from the first to the fifth free throw in all of the three throwing series. Organization and execution of a movement incorporate sending commands from the brain, with precise order and timing of motor units' activation. It is very important that commands have a proper organization, in order to have well coordinated, strong, and well-timed movement. Feedback has a major role in the process of execution of the complex movement. Performing five consecutive free throws, a player gets the feedback after each shot, that aid the adjustment of motor commands. For example, if the throw was too short, feedback will help the recruitment of more motor units for a stronger free throw. The comparison of visual information of the movement execution with kinesthetic information received from the movement performance would provide more accuracy, coordination, and optimal strength in the next attempt. A free throw lasts longer than a third of a second, feedback can help in control, and accuracy of movement performance (closed control loop). The speed of information processing is improving with the number of repetitions (Abernethy et al., 2012). Consequently, the results of the present study are in line with contemporary theoretical approaches. Secondly, the decline in successful shooting is apparent at the first free throw after the second stretching round (sixth and eleventh throw, Figure 2). Immediately after $15 \mathrm{~s}$ of static stretching of the triceps brachii muscle and flexor carpi radialis muscle, there was no difference in the percentage of successful free throws for $11 \%$ and $8 \%$ respectively. Previous static stretching of aforementioned muscles had a negative impact, although not statistically significant, on free throws shooting accuracy. However, as for the sixth to eighth free throws, the percentage of successful free throws has increased, and it could be said that influence weakens relatively quickly. To be more precise, that influence completely disappears about $15 \mathrm{~s}$ after the stretching of the triceps brachii muscle, and about $25 \mathrm{~s}$ after the stretching of the flexor carpi radialis muscle (Figure 2 - eighth and fifteenth free throw in comparison to the fifth free throw). Considering that numerous studies clearly demonstrated non-existence of long-lasting effects of the stretching exercises on the resistance of muscle-tendon unit (Björklund, Hamberg, \& Crenshaw, 2001; Halbertsma \& Göeken, 1994; Halbertsma, Mulder, Göeken, \& Eisma, 1999; Harvey et al., 2003; Magnusson, Aagard, Simonsen, \& BojsenMoller, 1998), it could be said that this result was to be expected. It seems that static stretching produces acute negative effects, not only for vertical jump and speed (Behm \& Chaouachi, 2011; Knudson, Bennett, Corn, Leick, \& Smith, 2001; Little \& Williams, 2006; Siatras, Papadopoulos, Mameletzi, Gerodimos, \& Kellis, 2003), but for the free throws accuracy as well. Some research reports have stated that static stretching techniques decrease neuromuscular sensitivity and that might be one of the reasons for the acute decrease in performance (Avela et al., 1999; Ribot-Ciscar et al., 1991; Vujnovich \& Dawson, 1994). Gergley (2009) found $31.04 \%$ decrease in full-swing golf shots accuracy after 20 minutes of total-body passive static stretching routine. A theoretical explanation for this acute decrease in performance included more compliant muscle-tendon unit, decreased neuromuscular reflex sensitivity, and neural inhibition that was attributed to the static stretching treatment.

Except the fact that the basketball players with above average training sessions per week had higher percentage of scored free throws $(72 \%>68 \%$; Figures 3 and 4), it is clear that their increasing trend of successful free throws is more regular. However, the MannWhitney $U$ test has shown there are no differences between the players with bellow and above average training sessions per week in any series of free throws [Asymp. sig. (2-tailed) $p>.05$ ]. It seems that difference in these percentages might be attributed to some other variables, not only to the stretching routines (for example break of execution of the motor task).

Furthermore, they had an increasing tendency of scored free throws after the stretching of flexor carpi radialis muscle. It is not that surprising to see this trend of improved accuracy as a function of practice, as participants practice throwing task they became better able to coadapt, the release speed and angle, in particular, to maintain an accurate outcome (Kudo, Tsutsui, Ishikura, Ito, \& Yamamoto, 2000).

It would be wrong to assume that the players with above average training sessions per week are simply better, therefor, more successful in performing free throws. Namely, the players with below the average training sessions per week had a better percentage of free throws before the stretching (70.6\%) than players with the above average training sessions per week (69.6\%; Figures 3 and 4, first 5 throws). Hence, the effects of the stretching, for the players with above average training sessions per week, diminish faster. A greater tolerance is achieved with frequent stretching exercises, so it is 
logical to see the advantage of the players with the above average training sessions per week.

As far as players' experience is concerned, it could be said that there is no influence of the static stretching of the triceps brachii and flexor carpi radialis muscles on the accuracy difference of free throws between experienced and less experienced players (Figures 5 and 6). However, analysis of the sixth and eleventh throws, which were the focus of this study, clearly indicates the negative influence of the prior stretching in both groups of basketball players. Such influence is more evident for players with less experience. In other words, it could be assumed that the muscle of the player with more experience has the ability of a quicker readjustment to the initial state (before the stretching). Anyhow, considering the differences in the free throw percentages are small, it is highly likely that some other factors had an impact on the differences between experienced and less experienced players.

It is worth noting that that simple break of activity or execution of the motor task might be one of the reasons for poorer performance, as well.

The lack of the apparatuses, which could level up the degree of the muscle stretching, could be one of the shortcomings of this study. Some future study could be based on the same concept, but with the dynamic stretching of the shooting arm. Furthermore, it could be useful to examine acute stretching of the arm muscles on the ball passing accuracy in basketball.

\section{Conclusion}

Fifteen seconds of static stretching of the triceps brachii and flexor carpi radialis muscles was performed just before the execution of free throw in basketball and it seems after that shooting accuracy have decreased. This decrease in the shooting accuracy is relatively short in duration (cca $15 \mathrm{~s}$ after the stretching of the triceps brachii muscle, and cca $25 \mathrm{~s}$ after the stretching of the flexor carpi radialis muscle), but basketball players can be advised not to perform stretching of the upper arm and/or forearm just before executing the free throws. This temporary decrease in shooting accuracy disappears faster in players with a higher number of training sessions per week, which could be explained by the better tolerance of these players to the performed stretching. Although it is the somewhat quicker recovery of the triceps brachii and flexor carpi radialis muscles of more experienced players to the initial state, it is presumed that some other factors (besides experience) had influenced their better free throw percentage after the stretching. Another thing that might cause poorer performance could be a simple break of activity or execution of the motor task.

The results of this research suggest that a static stretching routine of the upper arm and forearm muscles should be avoided before practice or competition.

\section{Acknowledgments}

The authors express their gratitude to the athletes and their coaches who participated in this study.

\section{Conflict of interest}

There were no conflicts of interest.

\section{References}

Abernethy, B., Kippers, V., Hanrahan, S. J., Pandy, M. G., McManus, A. M., \& Mackinnon, L. (2012). Biophysical foundations of human movement ( $3 \mathrm{rd}$ ed.). Champaign, IL: Human Kinetics.

Avela, J., Kyrolainen, H., \& Komi, P. (1999). Altered reflex sensitivity after repeated and prolonged passive muscle stretching. Journal of Applied Physiology, 86, 1283-1291.

Behm, D. G., \& Chaouachi, A. (2011). A review of the acute effects of static and dynamic stretching on performance. European Journal of Applied Physiology, 111, 2633-2651.

Bilodeau, E. A., Bilodeau, I. M., \& Schumsky, D. A. (1959). Some effects of introducing and withdrawing knowledge of results early and late in practice. Journal of Experimental Psychology, 58, 142-144.

Björklund, M., Hamberg, J., \& Crenshaw, A. G. (2001). Sensory adaptation after a 2-week stretching regimen of the rectus femoris muscle. Archives Physical Medicine and Rehabilitation, 82, 1245-1250.

Bošković, M. (1990). Anatomija čoveka [Human anatomy]. Belgrade, Zagreb, Yugoslavia: Medical book.

Button, C., Macleod, M., Sanders, R., \& Coleman, S. (2003). Examining movement variability in the basketball freethrow action at different skill levels. Research Quarterly for Exercise and Sport, 74, 257-269.

Chaouachi, A., Castagna, C., Chtara, M., Brughelli, M., Turki, O., Galy, O., ... Behm, D. G. (2010). Effect of warmups involving static or dynamic stretching on agility, sprinting and jumping performance in trained individuals. Journal of Strength and Conditioning Research, 24, 2001-2011.

Cramer, J. T., Housh, T. J., Johnson, G. O., Miller, J. M., Coburn, J. W., \& Beck, T. W. (2004). Acute effects of static stretching on peak torque in women. Journal of Strength and Conditioning Research, 18, 236-241.

Csataljay, G., O’Donoghue, P., Hughes, M., \& Dancs, H. (2009). Performance indicators that distinguish winning and losing teams in basketball. International Journal of Performance Analysis in Sport, 9, 60-66.

Foster, D., Weigand, D., \& Baines, D. (2006). The effect of removing superstitious behavior and introducing a 
pre-performance routine on basketball free-throw performance. Journal of Applied Sport Psychology, 18, 167-171.

Gergley, J. C. (2009). Acute effects of passive stretching during warm-up on driver clubhead speed, distance, accuracy, and consistent ball contact in young male competitive golfers. Journal of Strength and Conditioning Research, 23, 863-867.

Halbertsma, J. P., \& Göeken, L. N. (1994). Stretching exercises: Effect on passive extensibility and stiffness in short hamstrings of healthy subjects. Archives of Physical Medicine and Rehabilitation, 75, 976-981.

Halbertsma, J. P., Mulder, I., Göeken, L. N., \& Eisma, W. H. (1999). Repeated passive stretching: Acute effect on the passive muscle moment and extensibility of short hamstrings. Archives of Physical Medicine and Rehabilitation, 80, 407-414.

Harvey, L. A., Byak, A. J., Ostrovskaya, M., Glinsky, J., Katte, L., \& Herbert, R. D. (2003). Randomised trial of the effects of four weeks of daily stretch on extensibility of hamstring muscles in people with spinal cord injuries. Australian Journal of Physiotherapy, 49, 176-181.

Karalejić, M., \& Jakovljević, S. (2001). Osnove košarke [The basics of basketball]. Belgrade, Yugoslavia: Faculty of Sports and Physical Education.

Kay, A. D., \& Blazevich, A. J. (2008). Reductions in active plantar flexor moment are significantly correlated with static stretch duration. European Journal of Sport Science, 8, 41-46.

Knudson, D., Bennett, K., Corn, R., Leick, D., \& Smith, C. (2001). Acute effects of stretching are not evident in the kinematics of the vertical jump. Journal of Strength and Conditioning Research, 15, 98-101.

Knudson, D., \& Noffal, G. (2005). Time course of stretchinduced isometric strength deficits. European Journal of Applied Physiology, 94, 348-351.

Kudo, K., Tsutsui, S., Ishikura, T., Ito, T., \& Yamamoto, Y. (2000). Compensatory coordination of release parameters in a throwing task. Journal of Motor Behavior, 32, 337-345.

Little, T., \& Williams, A. G. (2006). Effects of differential stretching protocols during warm-ups on high-speed motor capacities in professional soccer players. Journal of Strength and Conditioning Research, 20, 203-307.

Magnusson, S. P., Aagard, P., Simonsen, E., \& Bojsen-Moller, F. (1998). A biomechanical evaluation of cyclic and static stretch in human skeletal muscle. International Journal of Sports Medicines, 19, 310-316.

Mah, C., Mah, K., Kezirian, E., \& Dement, W. (2011). The effects of sleep extension on the athletic performance of collegiate basketball players. Sleep, 34, 943-950.

Malacko, J. (2000). Osnove sportskog treninga [The basics of sport training]. Belgrade, Yugoslavia: Sports academy.

Memmert, D. (2006). Long-term effects of type of practice on the learning and transfer of a complex motor skill. Perceptual and Motor Skills, 103, 912-916.
Mullineaux, D., \& Uhl, T. (2010). Coordination-variability and kinematics of misses versus swishes of basketball free throws. Journal of Sports Sciences, 28, 1017-1024.

Navarro, R. M., Lorenzo, A., Gómez, M. A., \& Sampaio, J. (2009). Analysis of critical moments in the league ACB 2007-08. Revista de Psicologia del Deporte, 18, 391-395.

Perić, D., \& Petrović, B. (2015). Antropomotorika [Anthropomotorics]. Banja Luka, Bosnia and Herzegovina: Faculty of Physical Education and Sports.

Ribot-Ciscar, E., Tardy-Gervet, M., Vedel, J., \& Roll, J. (1991). Post-contraction changes in human muscle spindle resting discharge and stretch sensitivity. Experimental Brain Research, 86, 673-678.

Sampaio, J., Lorenzo, A., \& Ribeiro, C. (2006). Momentos críticos en los partidos de baloncesto: Metodología para identificación y análisis de los acontecimientos precedentes [Critical moments in basketball games: Methodology for identification and analysis of the events preceding]. Cultura, Ciencia y Deporte, 2(5), 83-88.

Schmidt, A. (2012). Movement pattern recognition in basketball free-throw shooting. Human Movement Science, 31, 360-382.

Siatras, T., Papadopoulos, G., Mameletzi, D., Gerodimos, V., \& Kellis, S. (2003). Static and dynamic acute stretching effect on gymnasts' speed in vaulting. Pediatric Exercise Science, 15, 383-391.

Stojanović, M. D., Stojanović, M. V., Veljović, D., Međedović, B., \& Ostojić, S. M. (2009). Uloga i značaj istezanja u sportu [The role and significance of stretching in sports]. TIMS Acta, 3, 66-72.

Vujnovich, A., \& Dawson, N. (1994). The effects of therapeutic muscle stretch on neural processing. Journal of Orthopaedic \& Sports Physical Therapy, 20, 145-153.

Weerapong, P., Hume, P. A., \& Kolt, G. S. (2004). Stretching: Mechanisms and benefits for sport performance and injury prevention. Physical Therapy Reviews, 9, 189-206.

Wilson, M., Vine, S., \& Wood, G. (2009). The influence of anxiety on visual attentional control in basketball free throw shooting. Journal of Sport \& Exercise Psychology, 31, 152-168.

Woolstenhulme, T., Bailey, B., \& Allsen, P. (2004). Vertical jump, anaerobic power, and shooting accuracy are not altered 6 hours after strength training in collegiate women basketball players. Journal of Strength and Conditioning Research, 18, 422-425.

Zachry, T., Wulf, G., Mercer, J., \& Bezodis, N. (2005). Increased movement accuracy and reduced EMG activity as the result of adopting an external focus of attention. Brain Research Bulletin, 67, 304-309.

Zuzik, P. (2011). Free throw shooting effectiveness in basketball matches of men and women. Sport Science Review, 20, 149-160. 